\title{
Calponin 3 suppresses proliferation, migration and invasion of non-small cell lung cancer cells
}

\author{
CHENGLIN YANG ${ }^{1}$, SHIPING ZHU ${ }^{1}$, WEIFENG FENG ${ }^{1}$ and XUEXIN CHEN ${ }^{2}$ \\ ${ }^{1}$ Traditional Chinese Medicine Department, The First Affiliated Hospital of Jinan University, Guangzhou, Guangdong 510632; \\ ${ }^{2}$ Department of Respiratory and Critical Care Medicine, The General Hospital of Yima Coal Industry Group Co. Ltd., \\ Yima, Henan 472300, P.R. China
}

Received January 8, 2021; Accepted May 28, 2021

DOI: $10.3892 / 01.2021 .12895$

\begin{abstract}
Calponin 3 (CNN3) is known to serve a role in certain types of cancer, such as gastric cancer and colorectal cancer. The present study investigated the clinical significance of CNN3 in non-small cell lung cancer (NSCLC) by evaluating its expression profile and relationship with disease prognosis using the Gene Expression Omnibus repository, Gene Expression Profiling Interactive Analysis 2 (GEPIA2) and Kaplan-Meier plotter analysis. CNN3 mRNA expression was measured using reverse transcription-quantitative PCR, while the protein expression level was measured using western blot analysis. Cell proliferation, cell cycle and apoptosis, and migration and invasion were analyzed using MTS assay, flow cytometry and Transwell assays, respectively. These results revealed that $\mathrm{CNN} 3 \mathrm{mRNA}$ expression was downregulated in NSCLC tissues compared with that in normal tissues. Additionally, CNN3 expression had a high diagnostic value based on the GSE2514 dataset and the data from The Cancer Genome Atlas and the Genotype Tissue Expression database, whereas it had a low diagnostic value based on the GSE10072 dataset. Furthermore, CNN3 expression was associated with survival in patients with lung adenocarcinoma (LUAD), whereas it was not associated with survival in patients with lung squamous cell carcinoma (LUSC) according to the Kaplan-Meier plotter results. According to the data from GEPIA2, and the GSE72094, GSE41271 and GSE31210 datasets, CNN3 expression was not associated with the prognosis of patients with LUAD and LUSC. The mRNA and protein expression levels of CNN3 were lower in two NSCLC cell lines (A549 and SK-MES-1) than in a human bronchial epithelial cell line (BEAS-2B). CNN3 overexpression suppressed cell
\end{abstract}

Correspondence to: Dr Chenglin Yang, Traditional Chinese Medicine Department, The First Affiliated Hospital of Jinan University, 613 Huangpu Avenue West, Tianhe, Guangzhou, Guangdong 510632, P.R. China

E-mail: y5804458@163.com

Key words: non-small cell lung cancer, calponin 3, proliferation, metastasis proliferation, migration and invasion, induced $\mathrm{G}_{1}$-phase arrest, promoted apoptosis and suppressed PI3K/AKT signaling pathway activation in the NSCLC cell lines, whereas CNN3 overexpression had no effect on cell morphology. In conclusion, CNN3 suppressed the proliferation and metastasis of NSCLC cells by downregulating the PI3K/AKT signaling pathway, making it a potential therapeutic target in this disease.

\section{Introduction}

Lung cancer is the most common of the major types of malignant diseases, presenting the greatest threat to the health and lives of individuals worldwide (1). Among the various types of cancer, lung cancer ranks first in terms of its incidence and mortality rate in men, whereas it ranks second for these parameters in women (1). An estimated 2.09 million individuals worldwide were newly diagnosed with lung cancer in 2018, the highest number among all types of cancer $(2,3)$. In China, the lung cancer incidence and mortality rate have also increased rapidly in recent years, attributable in large parts to smoking habits and severe air pollution (4). Although surgery is considered the most effective treatment for lung cancer (5), more than half of afflicted patients are already in an advanced metastatic stage of the disease at the time of diagnosis, which is beyond the optimal period for surgical intervention (6). Despite great improvements in therapeutic strategies, the 5-year survival rate of patients with lung cancer is still unsatisfactory (7). Therefore, the identification of novel and efficient molecular markers and clinical targets for the prevention and treatment of the disease remains a critical goal.

A previous study suggested that the occurrence and development of lung cancer are associated with the dysregulated expression and mutation of oncogenes and tumor suppressor genes (8), and a number of proteins and non-coding RNAs have been demonstrated to be involved in the pathogenesis of the disease $(9,10)$. Previous studies have demonstrated that Musashi 2 and fibroblast growth factor 19 act as independent prognostic biomarkers in non-small cell lung cancer (NSCLC) $(11,12)$. Mitotic spindle organizing protein 2A overexpression promotes NSCLC cell proliferation and invasion, which is associated with poor NSCLC prognosis (13). However, the pathological processes leading to lung cancer development are complex and still not fully 
understood. Therefore, further exploration of the functions and underlying mechanisms of other cancer-related genes is still essential. Calponin 3 (CNN3), a member of the calponin family comprising actin filament-associated proteins (14), acts as a regulator of actin cytoskeleton reorganization and dynamics (15). During embryonic development, CNN3 is expressed in the myoblasts and serves an important role in trophoblast fusion through its regulation of actin cytoskeleton rearrangement $(16,17)$. Furthermore, CNN3 expression is increased in cervical cancer cells, promoting the growth and metastasis of the disease (18). In gastric cancer, elevated CNN3 expression contributes to cancer cell resistance to doxorubicin (19). Additionally, CNN3 has been revealed to be a potential diagnostic marker of lymph node metastasis in colorectal cancer (20). In a more recent study, CNN3 was identified to be associated with a poor prognosis in patients with osteosarcoma, and could act as a potential prognostic biomarker (21). These aforementioned findings suggest that CNN3 serves various important roles in the pathogenesis of different types of cancer. However, to the best of our knowledge, its clinical significance and functions in NSCLC have not yet been investigated. Therefore, the present study aimed to explore the function of CNN3 in NSCLC and whether the protein could be used as a prognostic marker of this disease.

The present study focused on the role of CNN3 in NSCLC, including lung squamous cell carcinoma (LUSC), lung adenocarcinoma (LUAD) and large-cell lung carcinoma, which comprises all lung cancer types other than the small cell carcinomas (22). The relative expression levels of CNN3 in NSCLC and its association with disease prognosis were first analyzed using bioinformatics. Subsequently, the roles of CNN3 in NSCLC cell proliferation, migration and invasion were assessed. Finally, the mechanisms underlying the regulatory activity of CNN3 were investigated.

\section{Materials and methods}

Bioinformatics analysis. The GSE2514 (23), GSE10072 (24), GSE72094 (25), GSE41271 (26) and GSE31210 (27) datasets were selected on Gene Expression Omnibus (GEO; https://www. ncbi.nlm.nih.gov/geo/), and the basic clinical information of the patients is shown in Tables SI and SII. CNN3 expression in LUAD and LUSC tissues was analyzed using the GEO2R tools available on GEO, including the GSE2514 and GSE10072 datasets. CNN3 expression in LUAD and LUSC tissues was also analyzed on the Gene Expression Profiling Interactive Analysis 2 (GEPIA2; http://gepia2.cancer-pku.cn/\#index) bioinformatics website (28), which includes data from The Cancer Genome Atlas (TCGA; https://portal.gdc.cancer.gov/) and Genotype Tissue Expression (GTEx; https:/gtexportal. org/). Additionally, the diagnostic value of CNN3 was determined according to the data from the GSE2514 dataset, the GSE10072 dataset, and TCGA and GTEx. The prognostic value of CNN3 in LUAD and LUSC was assessed using the Kaplan-Meier plotter (http://www.kmplot.com), the GEPIA2 website, and the GSE72094, GSE41271 and GSE31210 datasets. The groups with high and low CNN3 expression were established according to the 'Auto select best cutoff' using Kaplan-Meier plotter. For the analysis of the prognostic value of CNN3 in LUAD, the follow-up period was restricted to
150 months to exclude the late crossover event when using Kaplan-Meier plotter analysis.

Cell culture and transfection. A total of two NSCLC cell lines (LUAD cells, A549; and LUSC cells, SK-MES-1) and a human bronchial epithelial cell line (BEAS-2B) were obtained from The Cell Bank of Type Culture Collection of The Chinese Academy of Sciences. Another three NSCLC cell lines (LUAD cells, PC9 and NCI-H1975; and LUSC cells, NCI-H520) were obtained from Procell Life Science \& Technology Co., Ltd. The culture and passage of these cells were performed according to the methods provided by the suppliers. The BEAS-2B cells were cultured in bronchial epithelial growth medium (Lonza Group, Ltd.). The A549 and PC3 cells were cultured in F-12K medium, while the NCI-H1975 and NCI-H520 cell lines were cultured in RPMI-1640 medium and the SK-MES-1 cell line was cultured in Eagle's minimum essential medium (all from Gibco; Thermo Fisher Scientific, Inc.). All media was supplemented with $10 \% \mathrm{FBS}, 100 \mathrm{U} / \mathrm{ml}$ penicillin and $100 \mu \mathrm{g} / \mathrm{ml}$ streptomycin (all from Gibco; Thermo Fisher Scientific, Inc.). All the cell lines were maintained at $37^{\circ} \mathrm{C}$ in a humidified incubator with $5 \% \mathrm{CO}_{2}$.

The CNN3-coding sequence, synthesized by Guangzhou IGE Biotechnology LTD, was cloned into the pcDNA 3.1 vector (Invitrogen; Thermo Fisher Scientific, Inc.) to construct a CNN3 overexpression recombinant plasmid, referred to as CNN3-OV. The pcDNA 3.1 empty vector, referred to as negative control (NC), was used as a control. A total of $5 \mu \mathrm{g}$ CNN3-OV and NC were transfected into the A549, SK-MES-1, NCI-H1975 and NCI-H520 cells using Lipofectamine ${ }^{\circledR} 2000$ (Invitrogen; Thermo Fisher Scientific, Inc.) at $37^{\circ} \mathrm{C}$ for $24 \mathrm{~h}$, then used for the subsequent experimentation. The images of cell morphology were captured under a light microscope (Olympus Corporation).

Reverse transcription-quantitative PCR (RT-qPCR). Total RNA was extracted from cells using TRIzol ${ }^{\circledR}$ reagent (Invitrogen; Thermo Fisher Scientific, Inc.). After its quantification, $1 \mu \mathrm{g}$ total RNA was reverse transcribed into first-strand cDNA using the PrimeScript ${ }^{\mathrm{TM}}$ II 1st Strand cDNA Synthesis Kit (Takara Biotechnology Co., Ltd.), at $30^{\circ} \mathrm{C}$ for $10 \mathrm{~min}, 42^{\circ} \mathrm{C}$ for $60 \mathrm{~min}$, then $85^{\circ} \mathrm{C}$ for $10 \mathrm{~min}$. PCR was performed using an ABI 7500 RT-PCR system (Applied Biosystems; Thermo Fisher Scientific, Inc.) and the SYBR ${ }^{\circledR}$ Premix Ex Taq ${ }^{\mathrm{TM}}$ Kit (Takara Biotechnology Co., Ltd.). The following thermocycling conditions were used: Initial denaturation at $95^{\circ} \mathrm{C}$ for $5 \mathrm{~min}$, followed by 40 cycles at $95^{\circ} \mathrm{C}$ for $15 \mathrm{sec}$ and $60^{\circ} \mathrm{C}$ for $32 \mathrm{sec}$. The primer sequences used were as follows: CNN3 forward, 5'-CCCAGAAAGGAATGAGTGTGT-3' and reverse, 5'-CTCGCCATGATACTCATCAG-3'; and 18S ribosomal RNA (18S rRNA) forward, 5'-CCTGGATACCGCAGCTAG GA-3' and reverse, 5'-GCGGCGCAATACGAATGCCCC-3'. The 18S rRNA sequence was used as an internal control to normalize CNN3 expression. The relative expression levels of CNN3 were calculated using the $2^{-\Delta \Delta C q}$ method (29). The experiment was performed in triplicate.

Cell proliferation assay. The CellTiter $96^{\circledR}$ AQueous One Solution Cell Proliferation Assay (MTS assay; Promega Corporation) was used to evaluate cell proliferation, which 
does not require the formazan product to be dissolved prior to measuring the absorbance. In brief, A549 and SK-MES-1 cells $\left(1 \times 10^{4}\right.$ cells/well in $\left.100 \mu \mathrm{l}\right)$ transfected with the CNN3-OV or $\mathrm{NC}$ recombinant plasmids for $24 \mathrm{~h}$ were seeded into 96 -well plates. After culture for 0, 24, 48 and 72 h, AQueous One Solution reagent $(10 \mu \mathrm{l})$ was added to the wells, and the cells were further incubated at $37^{\circ} \mathrm{C}$ for $2 \mathrm{~h}$. The optical density at $490 \mathrm{~nm}\left(\mathrm{OD}_{490 \mathrm{~nm}}\right)$ was determined using a microplate reader (Bio-Rad Laboratories, Inc.). The experiment was performed in triplicate.

Cell cycle analysis. A549 and SK-MES-1 cells transfected with the CNN3-OV or NC recombinant plasmids for $48 \mathrm{~h}$ were collected and washed twice. Subsequently, the cells were fixed in ice-cold $70 \%$ ethanol at $25^{\circ} \mathrm{C}$ for $2 \mathrm{~h}$. Next, the cells were washed twice and then stained according to the instructions of the PI Cell Cycle Detection kit (Nanjing KeyGen Biotech Co., Ltd.). Cell cycle distribution was assessed using a BD FACSCanto flow cytometer (BD Biosciences) and the cells were analyzed using ModFit LT v4.1 (Verity Software House). The experiment was performed in triplicate.

Cell apoptosis assay. A549 and SK-MES-1 cells transfected with the CNN3-ov or NC recombinant plasmids for $48 \mathrm{~h}$ were collected and washed twice with ice-cold PBS. Subsequently, the cells were resuspended and stained according to the instructions of the Annexin V-FITC/PI Apoptosis Detection kit (Nanjing KeyGen Biotech Co., Ltd.). The percentage of apoptotic cells was assessed using a BD FACSCanto flow cytometer (BD Biosciences) and cells were analyzed using FACSDiva v8.0 (BD Biosciences). This experiment was performed in triplicate.

Transwell migration and invasion assays. A549 or SK-MES-1 cells were transfected with the CNN3-OV or NC recombinant plasmids for $24 \mathrm{~h}$. Subsequently, $2 \times 10^{5}$ cells in $200 \mu \mathrm{l}$ serum-free medium were seeded into the upper chamber of the polycarbonate filter Transwell chamber ( $8-\mu \mathrm{m}$; BD Biosciences). The lower chamber was filled with culture medium containing 10\% FBS (Gibco; Thermo Fisher Scientific, Inc.). The Transwell chamber was then incubated at $37^{\circ} \mathrm{C}$ in a humidified incubator with $5 \% \mathrm{CO}_{2}$ for $48 \mathrm{~h}$. Next, the cells unable to pass through the filter were removed with a cotton swab. Finally, after washing with PBS, the filter membrane from the upper chamber was first fixed with precooled $4 \%$ paraformaldehyde at $25^{\circ} \mathrm{C}$ for $10 \mathrm{~min}$, then stained with crystal violet $(0.5 \%)$ at $25^{\circ} \mathrm{C}$ for $15 \mathrm{~min}$. Subsequently, the numbers of migratory or invasive cells were counted under a light microscope (Olympus Corporation). For the Transwell invasion assay, 8- $\mu \mathrm{m}$ polycarbonate filter Transwell chambers were coated at $37^{\circ} \mathrm{C}$ for 30 min with Matrigel (BD Biosciences) and all other protocol steps were the same as those for the Transwell migration assay. The experiment was repeated five times.

Western blotting. Total protein was extracted from the cells using RIPA lysis buffer with protease and phosphatase inhibitors (all from Beyotime Institute of Biotechnology). The protein concentration in the supernatant was quantified using a BCA assay (Beyotime Institute of Biotechnology). After quantification of the total protein, $30 \mu \mathrm{g}$ of each protein sample was separated using 10\% SDS-PAGE and then transferred onto polyvinylidene fluoride membranes. Subsequently, all membranes were blocked with $5 \%$ skimmed milk diluted in TBS. After the membranes were blocked at $37^{\circ} \mathrm{C}$ for $1 \mathrm{~h}$, they were incubated at $4^{\circ} \mathrm{C}$ for $12 \mathrm{~h}$ with the following primary antibodies: Anti-CNN3 (dilution, 1:1,000; cat. no. ab93593), anti-PI3K p85 $\alpha$ (dilution, 1:1,000; cat. no. ab191606), anti-phosphorylated AKT1 (p-AKT1 Ser473) (dilution, 1:5,000; cat. no. ab81283), anti-AKT1 (dilution, 1:1,000; cat. no. ab233755) and anti-GAPDH (dilution, 1:3,000; cat. no. ab181602) (all from Abcam). Subsequently, the membranes were incubated with HRP-conjugated secondary antibodies (both at dilution, 1:10,000) (cat. nos. ab205718 and ab6708) (both from Abcam) at $25^{\circ} \mathrm{C}$ for $40 \mathrm{~min}$. Finally, an enhanced chemiluminescence detection kit (Nanjing KeyGen Biotech Co., Ltd.) was used for visualization of the target proteins, and the ECL signal was exposed to X-rays. Densitometry of the bands was measured with ImageJ software (v6; National Institutes of Health). The experiment was repeated twice.

Statistical analysis. Statistical analysis was performed using SPSS 19.0 statistical software (IBM Corp.). Data are presented as the mean \pm standard deviation. The diagnostic value of CNN3 was determined using receiver operating characteristic (ROC) curve analysis, and then the cutoff value, sensitivity and specificity were calculated according to the Youden index. Survival curves were plotted using the Kaplan-Meier method and compared using the log-rank test. Differences between two groups were analyzed using an unpaired t-test. However, the differences in the CNN3 expression level between LUAD and normal paired adjacent lung tissues in the GSE2514 dataset were analyzed using a paired t-test. Differences in the CNN3 expression levels among the NSCLC and BEAS-2B cell lines were analyzed using one-way ANOVA followed by Dunnett's post hoc multiple comparison test. $\mathrm{P}<0.05$ was considered to indicate a statistically significant difference.

\section{Results}

Association between CNN3 expression and prognosis in patients with NSCLC. The analyses using the bioinformatics tools GEO and GEPIA2 revealed that the expression levels of CNN3 were significantly downregulated in both LUAD and LUSC tissues compared with in normal lung tissues (Fig. 1A and B, respectively). Additionally, the diagnostic value of CNN3 was determined using ROC curve analysis. The area under the curve values for CNN3 were 0.824 and 0.596 for LUAD according to the GSE2514 and GSE10072 datasets, respectively (Fig. S1A), whereas they were 0.830 for LUSC and 0.819 for LUAD according to data from TCGA and the GTEx project database (Fig. S1A). Therefore, except for the GSE10072 dataset, CNN3 was determined to have a high diagnostic value based on data from the GSE2514 dataset and the LUAD and LUSC data from TCGA and the GTEx database. The Kaplan-Meier plotter tool was used to investigate the prognostic potential of CNN3 in NSCLC and the results revealed that low $\mathrm{CNN} 3$ expression was associated with a shorter survival time for LUAD, while it exhibited no association for patients with LUSC (Fig. 1C). However, CNN3 expression was not associated with the survival rate of patients 
A

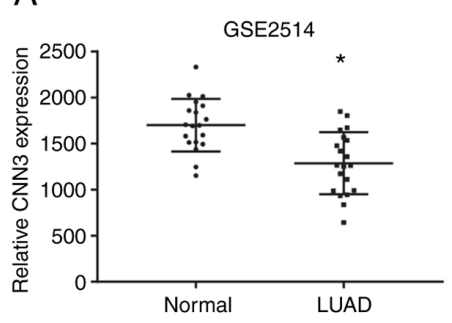

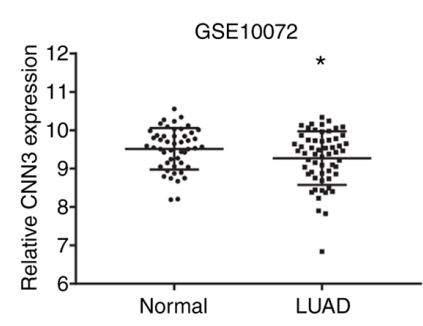

B

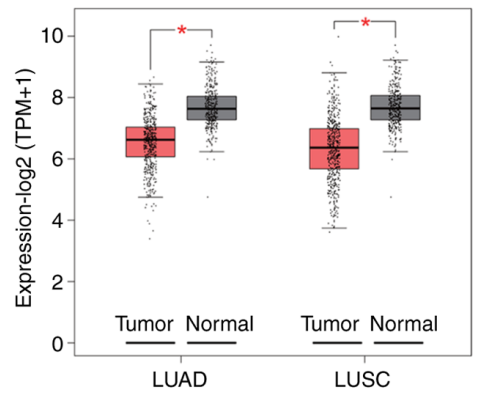

C

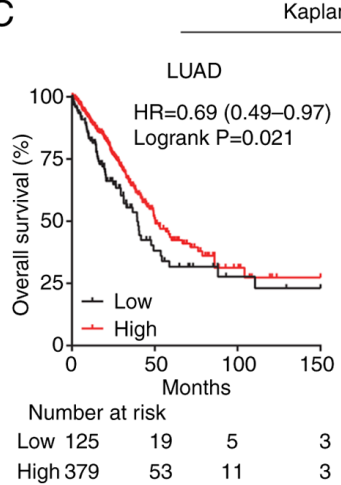

Kaplan-Meier plotter

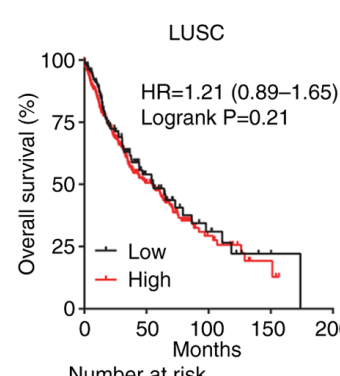

Number at risk

Low $\begin{array}{llll}135 & 30 & 9\end{array}$

High $\begin{array}{llll}360 & 75 & 18 & 3\end{array}$

D

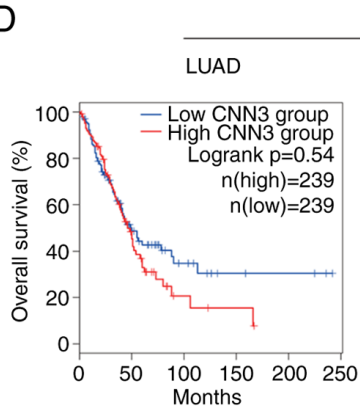

GEPIA2

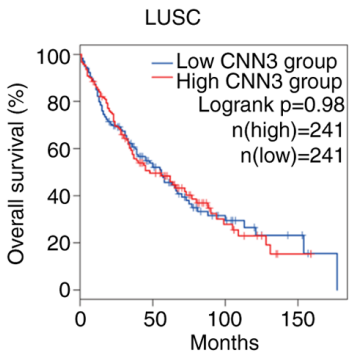

Figure 1. Association between CNN3 expression and the prognosis of patients with NSCLC. (A) CNN3 expression in LUAD and normal lung tissues was assessed using the GSE2514 and GSE10072 datasets from the Gene Expression Omnibus database. Normal lung tissues in GSE2514 dataset were paired adjacent tissues and in the GSE10072 dataset obtained from different individuals. (B) Expression levels of CNN3 in LUAD and LUSC tumor tissues and normal lung tissues (obtained from different individuals) were assessed using the bioinformatics tool GEPIA2. (C) Prognostic potential of CNN3 expression in NSCLC was evaluated using the Kaplan-Meier plotter. To analyze the prognostic value of CNN3 in LUAD, the follow-up period was restricted to 150 months to exclude the late crossover event when using Kaplan-Meier plotter analysis. (D) Prognostic potential of CNN3 expression in NSCLC was evaluated using GEPIA2. * $\mathrm{P}<0.05$. CNN3, calponin 3; GEPIA2, Gene Expression Profiling Interactive Analysis 2; HR, hazard ratio; LUAD, lung adenocarcinoma; LUSC, lung squamous cell carcinoma; NSCLC, non-small cell lung cancer; TPM, transcripts per million.
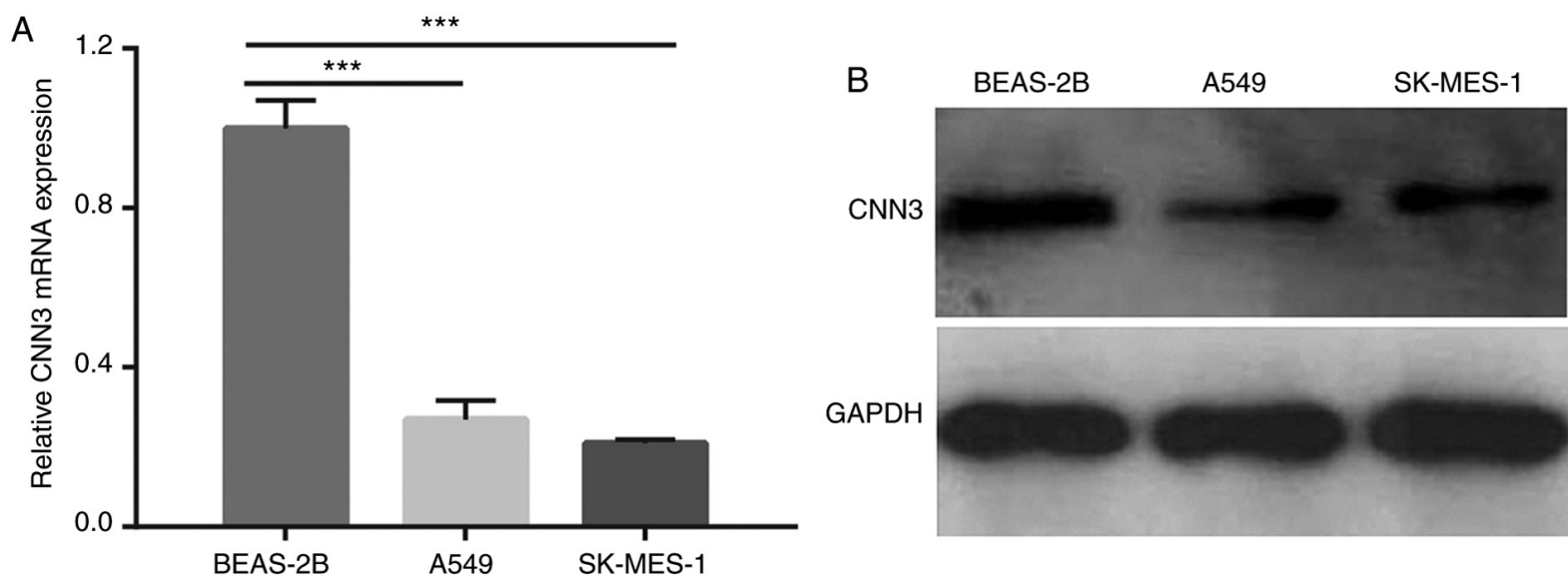

Figure 2. CNN3 expression in the A549 and SK-MES-1 cell lines was lower than that in BEAS-2B normal epithelial cells. (A) mRNA and (B) protein expression levels of CNN3 were examined by reverse transcription-quantitative PCR and western blotting, respectively. ${ }^{* * *} \mathrm{P}<0.001$. CNN3, calponin 3 .

with LUAD and LUSC according to GEPIA2 (Fig. 1D). In addition, CNN3 expression was also not associated with the prognosis of patients according to data from GEO (GSE72094, GSE41271 and GSE31210 datasets; Fig. S1B).

CNN3 expression is downregulated in NSCLC cell lines. The mRNA and protein expression levels of CNN3 in NSCLC cells were assessed by RT-qPCR and western blotting, respectively. The results demonstrated that both the mRNA and protein expression levels of CNN3 were notably downregulated in the A549 and SK-MES-1 cells compared with in BEAS-2B cells (Fig. 2A and B). Additionally, CNN3 mRNA expression was significantly downregulated in NCI-H1975, PC9 and NCI-H520 cells compared with in BEAS-2B cells (Fig. S2).

CNN3 was successfully overexpressed in NSCLC cell lines. The expression levels of CNN3 in A549 and SK-MES-1 cells, which were transfected with the CNN3-OV or NC recombinant plasmids for $48 \mathrm{~h}$, were measured using RT-qPCR and 

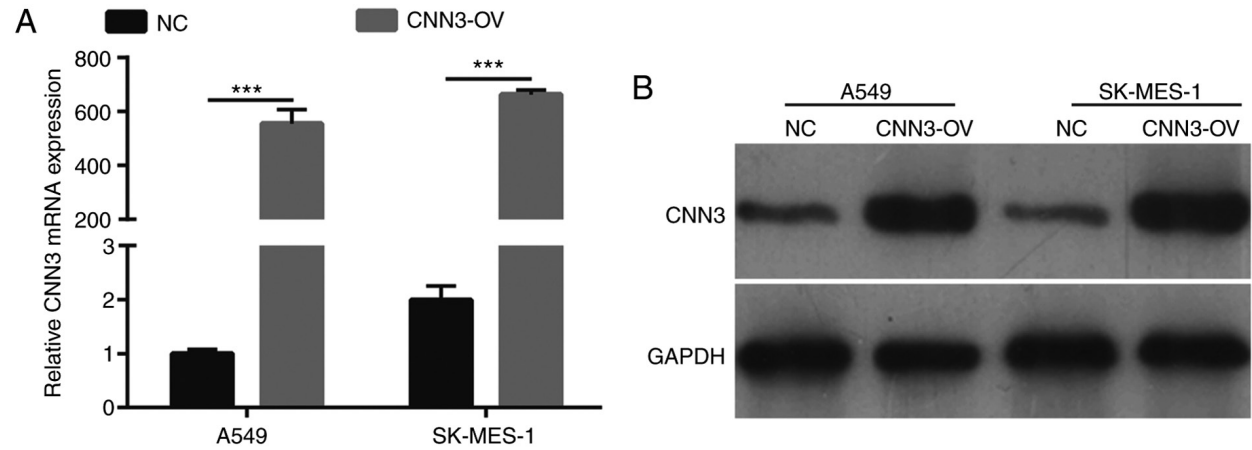

Figure 3. CNN3 mRNA and protein expression in A549 and SK-MES-1 cells transfected with the CNN3 overexpression recombinant plasmid (CNN3-OV) or NC. (A) CNN3 mRNA expression was measured by reverse transcription-quantitative PCR. ${ }^{* * *} \mathrm{P}<0.001$. (B) CNN3 protein expression was examined by western blotting. CNN3, calponin 3; NC, empty vector pcDNA 3.1 .

western blotting. In both cell lines, CNN3 mRNA and protein expression was notably higher in the CNN3-OV group than in the NC group (Fig. 3A and B).

CNN3 overexpression suppresses NSCLC cell proliferation and promotes apoptosis. To investigate the role of $\mathrm{CNN} 3$ in the pathogenesis of NSCLC, the present study analyzed the effects of its overexpression on proliferation, the cell cycle and apoptosis in both A549 and SK-MES-1 cells. As shown in Fig. $4 \mathrm{~A}$ and $\mathrm{B}$, in both cell lines, the $\mathrm{OD}_{490 \mathrm{~nm}}$ value was significantly lower in the CNN3-OV group than in the NC group after culture for 48 and $72 \mathrm{~h}$. Additionally, compared with that in the $\mathrm{NC}$ group, the percentage of cells in the $\mathrm{G}_{1}$ phase was higher, whereas that in the $\mathrm{S}$ phase was lower, in the CNN3-OV group (Fig. 4C). Furthermore, compared with that in the NC group the number of apoptotic cells in the CNN3-OV group was also high: 3.60 to $21.23 \%$ and 6.07 to $13.80 \%$ for the A549 and SK-MES-1 cell lines, respectively (Fig. 4D). These results indicated that $\mathrm{CNN} 3$ overexpression could suppress proliferation, induce $\mathrm{G}_{1}$-phase arrest and promote apoptosis in NSCLC cells. Additionally, CNN3 mRNA and protein expression level was notably higher in the CNN3-OV group compared with that in the NC group in both the NCI-H1975 and NCI-H520 cell lines (Fig. S3A and B). In both the NCI-H1975 and NCI-H520 cell lines, cell proliferation was significantly lower in the CNN3-OV group than in the NC group after culture for 24, 48 and $72 \mathrm{~h}$ (Fig. S3C and D).

CNN3 overexpression suppresses NSCLC cell migration and invasion. Next, the cell morphology was not markedly altered between the $\mathrm{NC}$ and $\mathrm{CNN} 3-\mathrm{OV}$ groups according to the results of light microscope and Transwell analysis (Fig. 5A and B). To investigate the effect of CNN3 overexpression on cell migration and invasion, Transwell migration and invasion assays were performed using the transfected A549 and SK-MES-1 cells. In both NSCLC cell lines, the CNN3-OV group had a lower number of migratory and invasive cells than the NC group (Fig. 5B). The inhibitory effect of CNN3 overexpression on A549 and SK-MES-1 cell migration was 33.00 and $36.58 \%$, respectively. The inhibitory effect of CNN3 overexpression on A549 and SK-MES-1 cell invasion was 39.28 and $41.52 \%$, respectively. These results suggested that CNN3 overexpression could suppress NSCLC cell migration and invasion.
CNN3 overexpression suppresses the PI3K/AKT signaling pathway in NSCLC cells. Western blot results revealed that the levels of PI3K p85 $\alpha$ and the ratio of p-AKT1/AKT1 were lower in the CNN3-OV group than in the NC group (Fig. 6). This suggested that $\mathrm{CNN} 3$ overexpression could suppress the PI3K/AKT signaling pathway in NSCLC cells.

\section{Discussion}

In the present study, bioinformatics analysis revealed that CNN3 expression was significantly downregulated in both LUAD and LUSC tissues compared with its expression in normal lung tissue, suggesting that $\mathrm{CNN} 3$ dysregulation may serve a key regulatory role in the pathogenesis of NSCLC. Therefore, the present study verified this hypothesis by investigating the effect of $\mathrm{CNN} 3$ overexpression on cell proliferation, migration, invasion and apoptosis, and the cell cycle distribution of A549 and SK-MES-1 NSCLC cell lines.

The present study revealed that CNN3 expression was significantly downregulated in both LUAD and LUSC tissues. However, the identified profile of downregulated $\mathrm{CNN} 3$ expression in NSCLC tissues was inconsistent with the expression profile in osteosarcoma and cervical cancer tissues $(18,21)$, which suggests that $\mathrm{CNN} 3$ may serve different roles in different types of cancer. Additionally, CNN3 expression had a high diagnostic value based on the GSE2514 dataset and the LUAD and LUSC data from TCGA and the GTEx database, whereas it had a low diagnostic value when based on the GSE10072 dataset. Furthermore, CNN3 expression was associated with overall survival rate in patients with LUAD, while it was not associated with overall survival rate in patients with LUSC according to the Kaplan-Meier plotter results. According to the data from TCGA, and the GSE72094, GSE41271 and GSE31210 datasets, CNN3 expression was not associated with the prognosis of patients with LUAD and LUSC. These results implied that CNN3 could not act as prognostic marker for monitoring patients with NSCLC, specifically for improving prognostic assessment in LUSC. The aforementioned results indicated that whether $\mathrm{CNN} 3$ can be used as a marker for diagnosis and prognosis of NSCLC requires further collection of NSCLC specimens for research.

The present study also demonstrated that the overexpression of CNN3 suppressed proliferation, migration and invasion in NSCLC cells. These in vitro results indicated that $\mathrm{CNN} 3$ may 

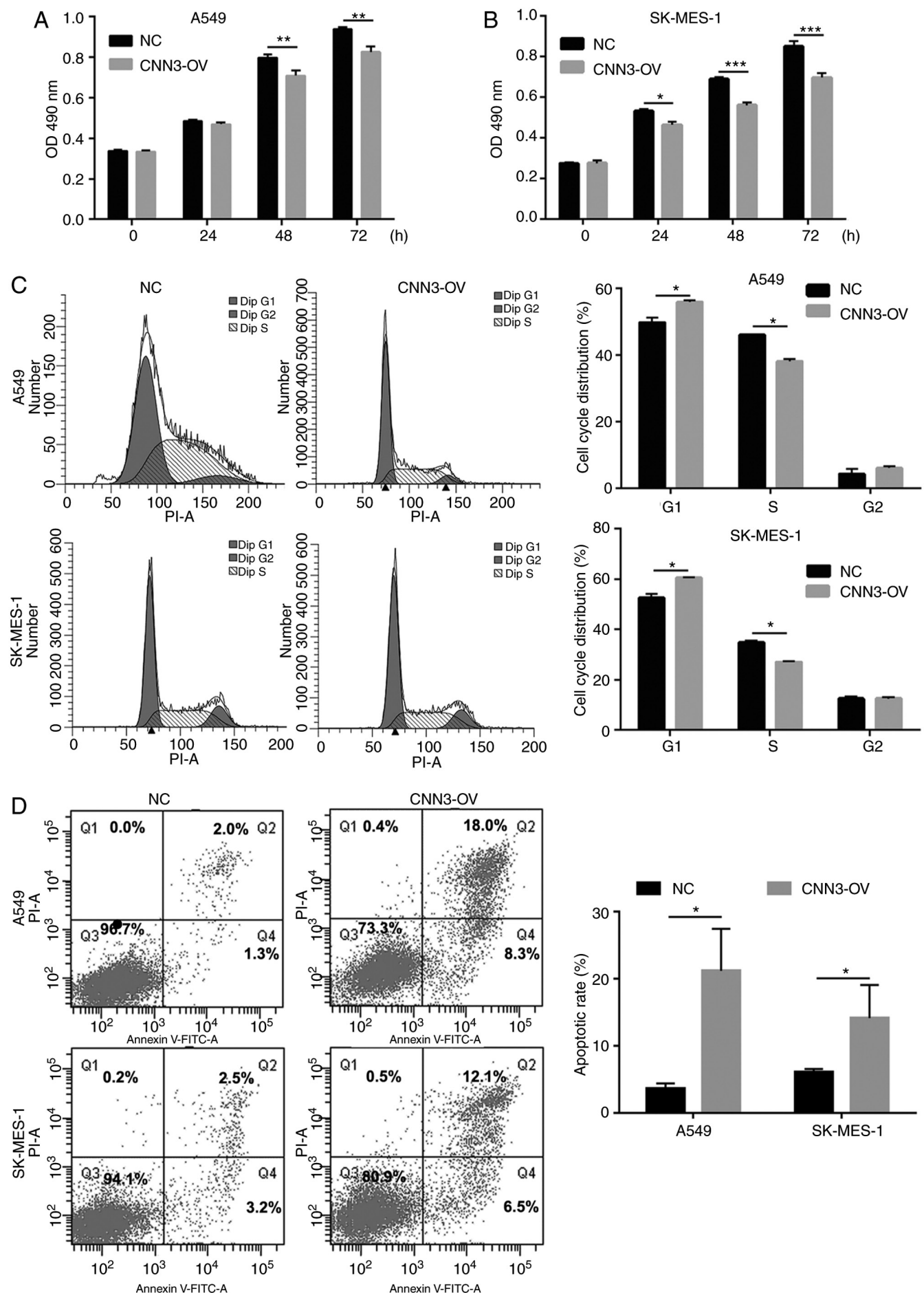

Figure 4. CNN3 overexpression suppresses cell proliferation and promotes apoptosis. A549 and SK-MES-1 cells were transfected with the CNN3 overexpression recombinant plasmid (CNN3-OV) or NC, and were referred to as $\mathrm{NC}$ and CNN3-OV, respectively. Effects of CNN3 overexpression on (A) A549 and (B) SK-MES-1 cell proliferation were measured using an MTS assay. ${ }^{*} \mathrm{P}<0.05,{ }^{* *} \mathrm{P}<0.01$ and ${ }^{* * * *} \mathrm{P}<0.001$. The effects of CNN3 overexpression on (C) the cell cycle and (D) apoptosis were assessed by flow cytometry. "P<0.05. CNN3, calponin 3; NC, empty vector pcDNA 3.1; OD, optical density.

serve a tumor-suppressive role in NSCLC. Although significant apoptosis occurred in the CNN3-overexpressing cells, the inhibitory effect of CNN3 overexpression on cell migration and invasion was greater than its effect on cell apoptosis. The results of the in vitro cell function assay in the present study were also supported by the present findings that patients with LUAD in the high CNN3 expression group had a longer survival time than those in the low CNN3 expression group, according to Kaplan-Meier plotter results. CNN3 has been reported to serve a tumor-promoting role in osteosarcoma, gastric cancer and colon cancer $(19,21,30)$. Actin cytoskeleton reorganization promotes cancer metastasis (31). CNN3 is one of the actin filament-associated proteins and functions to reorganize the actin cytoskeleton (14), suggesting that CNN3 may enhance the metastasis of cancer cells by promoting actin cytoskeleton reorganization in osteosarcoma, gastric cancer and colon cancer. However, no studies have demonstrated that there is an association between the effect of $\mathrm{CNN} 3$ on cell proliferation 

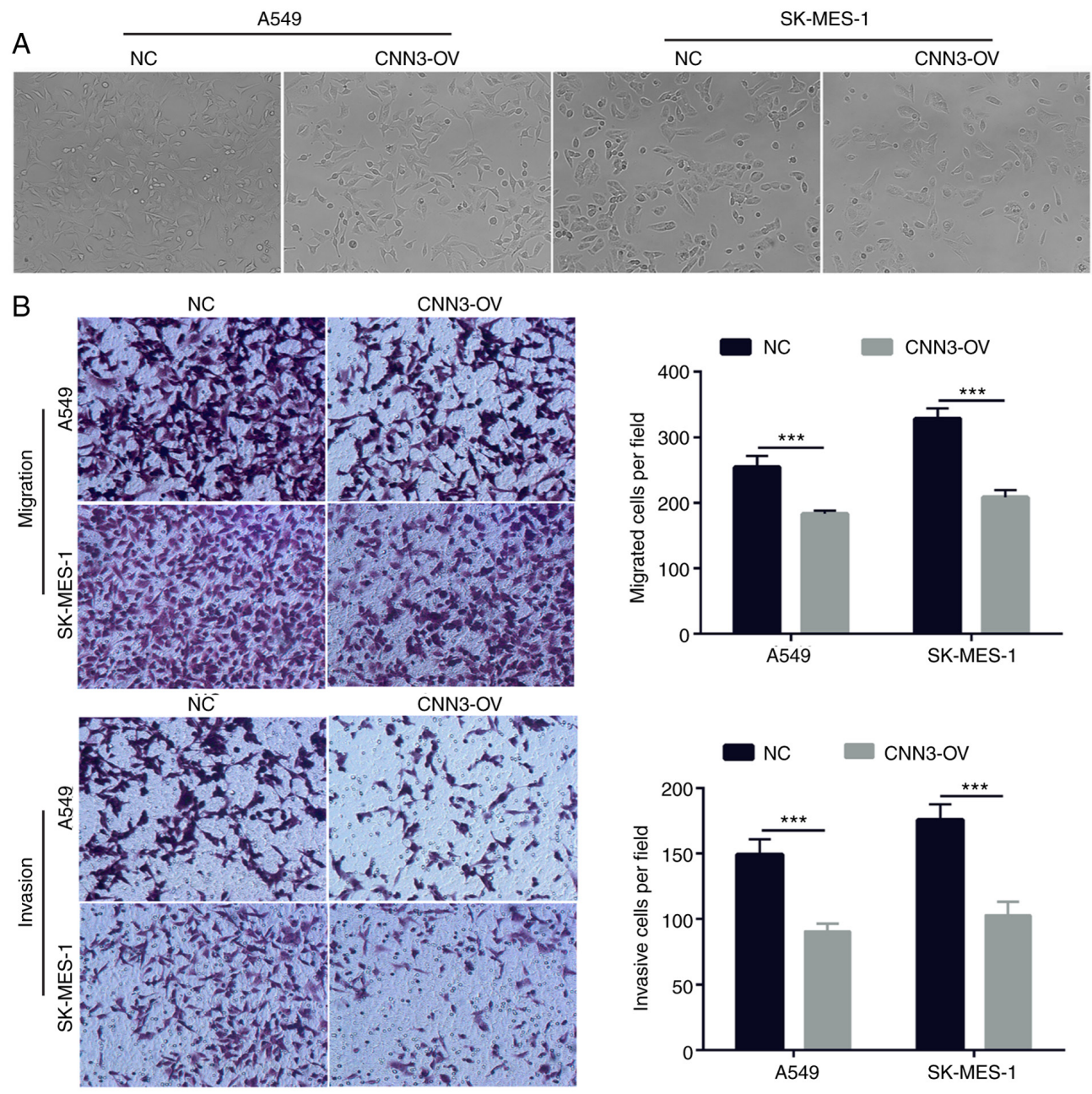

Figure 5. CNN3 overexpression suppresses cell migration and invasion but does not affect the cell morphology. A549 and SK-MES-1 cells were transfected with the CNN3 overexpression recombinant plasmid (CNN3-OV) or NC, and were referred to as NC and CNN3-OV, respectively. (A) Morphology images of the cells were captured under a light microscope. (B) Effects of CNN3 overexpression on cell migration and invasion were assessed using Transwell assays. Magnification, $\mathrm{x} 100 .{ }^{* * *} \mathrm{P}<0.001$. CNN3, calponin 3; NC, empty vector pcDNA 3.1.
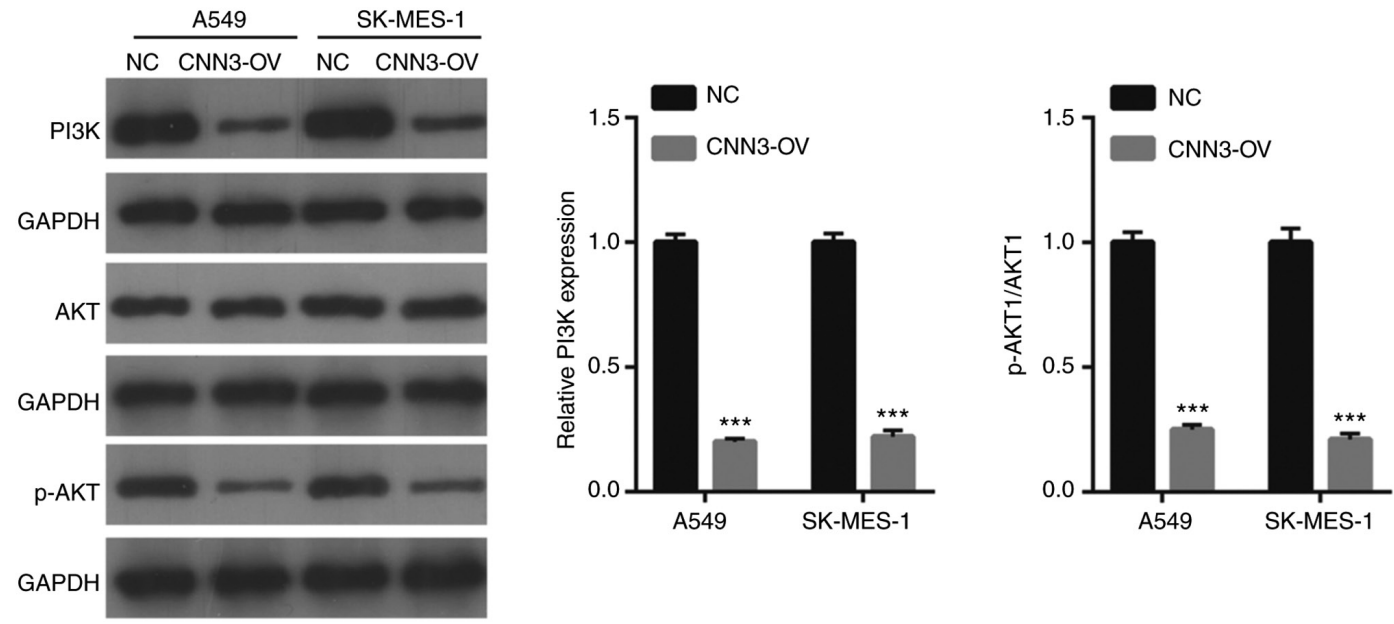

Figure 6. CNN3 overexpression inactivates the PI3K/AKT signaling pathway. A549 and SK-MES-1 cells were transfected with NC or the CNN3 overexpression recombinant plasmid (CNN3-OV), and were referred to as NC and CNN3-OV, respectively. The levels of PI3K p85 $\alpha$, p-AKT1 and AKT1 were examined by western blotting. ${ }^{* * *} \mathrm{P}<0.001$. CNN3, calponin 3 ; NC, empty vector pcDNA 3.1 ; p-, phosphorylated.

and migration and the changes in cell morphology. The present study revealed that CNN3 expression was lower in LUSC and LUAD tissues and cell lines, and CNN3 overexpression had no effect on the cell morphology, suggesting that CNN3 did not affect the proliferation and metastasis of cancer cells by promoting actin cytoskeleton reorganization. These results 
further support the hypothesis that CNN3 may serve different roles in different cancer types.

To investigate the regulatory mechanism underlying the tumor-suppressive role of CNN3 in NSCLC, the present study investigated the effect of CNN3 overexpression on the PI3K/AKT signaling pathway, the abnormal activation of which is known to be involved in numerous human diseases, including tumorigenesis (32-34). It was revealed that the overexpression of CNN3 decreased the levels of PI3K p85 $\alpha$ and the ratio of p-AKT1/AKT1 in A549 and SK-MES-1 cells. Human PI3K comprises a $110 \mathrm{kDa}$ catalytic subunit and a $85 \mathrm{kDa}$ regulatory subunit, the latter of which is PI3K p85 $\alpha$, also known as phosphoinositide 3-kinase regulatory subunit 1 (35). A total of 3 AKT isomers (AKT1, AKT2 and AKT3) mediate numerous downstream signaling pathways regulated by PI3K, which phosphorylates AKT at Ser473 (36,37). PI3K overexpression mediates downstream AKT phosphorylation, while not affecting the expression of total Akt protein $(36,37)$. Therefore, the present results demonstrated that the overexpression of CNN3 could inactivate the PI3K/AKT signaling pathway. The activation of this signaling pathway promotes the growth and metastasis of a number of cancer types, including NSCLC $(34,38)$. Given that CNN3 had suppressive effects on NSCLC cell proliferation, migration and invasion, it was hypothesized that the effects of this actin filament-associated protein in NSCLC cells may be mediated through its inhibition of the PI3K/AKT signaling pathway. To the best of our knowledge, the present study was the first to report that CNN3 is involved in the regulation of this important signaling pathway.

The present study had certain limitations. First, the diagnostic and prognostic functions of $\mathrm{CNN} 3$ were analyzed using GEO and TCGA data, leading to differences in results. Therefore, a large number of NSCLC tissues should be collected and follow-up analyses should be conducted to verify the possibility of its use as a prognostic and diagnostic marker for patients with NSCLC in China or worldwide. Additionally, the effect of CNN3 overexpression on the proliferation and metastasis of NSCLC cells still needs to be verified in in vivo experiments. Finally, the present study only examined the effect of CNN3 overexpression on the PI3K/AKT signaling pathway, and whether it can affect other signaling pathways has not been studied. Therefore, further experiments are required to analyze the role of CNN3 in the diagnosis and prognosis of NSCLC and the regulatory mechanism of CNN3 in the growth and metastasis of NSCLC.

In conclusion, the results revealed that $\mathrm{CNN} 3$ had a downregulated expression profile in NSCLC tissues and cells. CNN3 overexpression suppressed NSCLC cell proliferation, migration and invasion in vitro. Therefore, CNN3 may be a potential therapeutic target for NSCLC. However, whether CNN3 can be used as a diagnostic and prognostic marker remains to be studied further.

\section{Acknowledgements}

Not applicable.

\section{Funding}

No funding was received.

\section{Availability of data and materials}

The datasets used and/or analyzed during the current study are available from the corresponding author on reasonable request.

\section{Authors' contributions}

CY conceived and designed the present study, developed the methodology, and drafted the manuscript. CY and SZ performed the experiments and collected the data. WF and XC analyzed and interpreted the data. SZ, WF and XC revised the manuscript. All authors read and approved the final manuscript. CY and XC confirm the authenticity of all the raw data.

\section{Ethics approval and consent to participate}

Not applicable.

\section{Patient consent for publication}

Not applicable.

\section{Competing interests}

The authors declare that they have no competing interests.

\section{References}

1. Bray F, Ferlay J, Soerjomataram I, Siegel RL, Torre LA and Jemal A: Global cancer statistics 2018: GLOBOCAN estimates of incidence and mortality worldwide for 36 cancers in 185 countries. Ca Cancer J Clin 68: 394-424, 2018.

2. Siegel RL, Miller KD and Jemal A: Cancer statistics, 2019. CA Cancer J Clin 69: 7-34, 2019.

3. Ferlay J, Colombet M, Soerjomataram I, Dyba T, Randi G, Bettio M, Gavin A, Visser O and Bray F: Cancer incidence and mortality patterns in Europe: Estimates for 40 countries and 25 major cancers in 2018. Eur J Cancer 103: 356-387, 2018.

4. Cao M and Chen W: Epidemiology of lung cancer in China. Thorac Cancer 10: 3-7, 2019.

5. Richard PJ and Rengan R: Oligometastatic non-small-cell lung cancer: Current treatment strategies. Lung Cancer (Auckl) 7: 129-140, 2016.

6. Fischer C, Leithner K, Wohlkoenig C, Quehenberger F, Bertsch A, Olschewski A, Olschewski $\mathrm{H}$ and Hrzenjak A: Panobinostat reduces hypoxia-induced cisplatin resistance of non-small cell lung carcinoma cells via HIF-1 $\alpha$ destabilization. Mol Cancer 14: 4, 2015 .

7. Lakshmi SP, Reddy AT, Banno A and Reddy RC: PPAR agonists for the prevention and treatment of lung cancer. Ppar Res 2017: 8252796, 2017.

8. Mohamad N, Jayalakshmi P, Rhodes A, Liam CK, Tan JL and Yousoof S: Anaplastic lymphoma kinase (ALK) mutations in patients with adenocarcinoma of the lung. Br J Biomed Sci 74: 176-180, 2017.

9. Virmani AK and Gazdar AF: Tumor suppressor genes in lung cancer. Methods Mol Biol 222: 97-115, 2003.

10. Ghafouri-Fard S, Shoorei H, Branicki W and Taheri M: Non-coding RNA profile in lung cancer. Exp Mol Pathol 114: 104411, 2020.

11. Topchu I, Karnaukhov N, Mazitova A, Yugai V, Voloshin M, Tikhomirova M, Kit O, Frantsiyants E, Kharin L, Airapetova T, et al: Musashi 2 (MSI2) expression as an independent prognostic biomarker in non-small cell lung cancer (NSCLC). J Thorac Dis 13: 1370-1379, 2021.

12. Chen J, Shao J, Shen A, Zhu X, Zhang X, Sun H, Wei S and Ling Y: Enhanced expression of FGF19 predicts poor prognosis in patients with non-small cell lung cancer. J Thorac Dis 13: 1769-1784, 2021. 
13. Wang H, Jiang X, Cheng Y, Ren H, Hu Y, Zhang Y, Su H, Zou Z Wang Q, Liu Z, et al: MZT2A promotes NSCLC viability and invasion by increasing Akt phosphorylation via the MOZART2 domain. Cancer Sci 112: 2210-2222, 2021.

14. Takahashi K and Nadal-Ginard B: Molecular cloning and sequence analysis of smooth muscle calponin. J Biol Chem 266: 13284-13288, 1991.

15. Rami G, Caillard O, Medina I, Pellegrino C, Fattoum A, Ben-Ari Y and Ferhat L: Change in the shape and density of dendritic spines caused by overexpression of acidic calponin in cultured hippocampal neurons. Hippocampus 16: 183-197, 2006

16. Daimon E, Shibukawa Y and Wada Y: Calponin 3 regulates stress fiber formation in dermal fibroblasts during wound healing. Arch Dermatol Res 305: 571-584, 2013.

17. Shibukawa Y, Yamazaki N, Daimon E and Wada Y: Rock-dependent calponin 3 phosphorylation regulates myoblast fusion. Exp Cell Res 319: 633-648, 2013.

18. Xia L, Yue Y, Li M, Zhang YN, Zhao L, Lu W, Wang X and $\mathrm{Xie} \mathrm{X}$ : CNN3 acts as a potential oncogene in cervical cancer by affecting RPLP1 mRNA expression. Sci Rep 10: 2427, 2020.

19. Hong KS, Kim H, Kim SH, Kim M and Yoo J: Calponin 3 regulates cell invasion and doxorubicin resistance in gastric cancer Gastroenterol Res Pract 2019: 3024970, 2019.

20. Nakarai C, Osawa K, Akiyama M, Matsubara N, Ikeuchi H, Yamano T, Hirota S, Tomita N, Usami M and Kido Y: Expression of AKR1C3 and CNN3 as markers for detection of lymph node metastases in colorectal cancer. Clin Exp Med 15: 333-341, 2015

21. Dai F, Luo F, Zhou R, Zhou Q, Xu J, Zhang Z, Xiao J and Song L: Calponin 3 is associated with poor prognosis and regulates proliferation and metastasis in osteosarcoma. Aging (Albany NY) 12: 14037-14049, 2020.

22. Herbst RS, Morgensztern D and Boshoff C: The biology and management of non-small cell lung cancer. Nature 553: 446-454, 2018.

23. Stearman RS, Dwyer-Nield L, Zerbe L, Blaine SA, Chan Z, Bunn PA Jr,Johnson GL,Hirsch FR, MerrickDT,FranklinWA, etal: Analysis of orthologous gene expression between human pulmonary adenocarcinoma and a carcinogen-induced murine model. Am J Pathol 167: 1763-1775, 2005.

24. Landi MT, Dracheva T, Rotunno M, Figueroa JD, Liu H, Dasgupta A, Mann FE, Fukuoka J, Hames M, Bergen AW, et al: Gene expression signature of cigarette smoking and its role in lung adenocarcinoma development and survival. PLoS One 3: e1651,2008.

25. Schabath MB, WelshEA,Fulp WJ, Chen L, Teer JK, Thompson ZJ, Engel BE, Xie M, Berglund AE, Creelan BC, et al: Differential association of STK11 and TP53 with KRAS mutation-associated gene expression, proliferation and immune surveillance in lung adenocarcinoma. Oncogene 35: 3209-3216, 2016.
26. Sato M, Larsen JE, Lee W, Sun H, Shames DS, Dalvi MP, Ramirez RD, Tang H, DiMaio JM, Gao B, et al: Human lung epithelial cells progressed to malignancy through specific oncogenic manipulations. Mol Cancer Res 11: 638-650, 2013.

27. Okayama H, Kohno T, Ishii Y, Shimada Y, Shiraishi K, Iwakawa R, Furuta K, Tsuta K, Shibata T, Yamamoto S, et al: Identification of genes upregulated in ALK-positive and EGFR/KRAS/ALK-negative lung adenocarcinomas. Cancer Res 72: 100-111, 2012.

28. Tang Z, Kang B, Li C, Chen T and Zhang Z: GEPIA2: An enhanced web server for large-scale expression profiling and interactive analysis. Nucleic Acids Res 47: W556-W560, 2019.

29. Livak KJ and Schmittgen TD: Analysis of relative gene expression data using real-time quantitative PCR and the 2(-Delta Delta C(T)) method. Methods 25: 402-408, 2001.

30. Nair VA,Al-Khayyal NA, SivaperumalS and Abdel-Rahman WM: Calponin 3 promotes invasion and drug resistance of colon cancer cells. World J Gastrointest Oncol 11: 971-982, 2019.

31. Jiang X, Qin Y, Kun L and Zhou Y: The significant role of the microfilament system in tumors. Front Oncol 11: 620390, 2021.

32. Ediriweera MK, Tennekoon KH and Samarakoon SR: Role of the PI3K/AKT/mTOR signaling pathway in ovarian cancer: Biological and therapeutic significance. Semin Cancer Biol 59: 147-160, 2019.

33. Zhang X, He X, Liu Y, Zhang H, Chen H, Guo S and Liang Y. MiR-101-3p inhibits the growth and metastasis of non-small cell lung cancer through blocking PI3K/AKT signal pathway by targeting MALAT-1. Biomed Pharmacother 93: 1065-1073, 2017.

34. Chen H, Zhou L, Wu X, Li R, Wen J, Sha J and Wen X: The PI3K/AKT pathway in the pathogenesis of prostate cancer. Front Biosci (Landmark Ed) 21: 1084-1091, 2016.

35. Martini M, De Santis MC, Braccini L, Gulluni F and Hirsch E: PI3K/AKT signaling pathway and cancer: An updated review. Ann Med 46: 372-383, 2014.

36. Yang J, Cron P, Thompson V, Good VM, Hess D, Hemmings BA and Barford D: Molecular mechanism for the regulation of protein kinase B/Akt by hydrophobic motif phosphorylation. Mol Cell 9: 1227-1240, 2002.

37. Calera MR, Martinez C, Liu H, Jack AK, Birnbaum MJ and Pilch PF: Insulin increases the association of Akt-2 with Glut4-containing vesicles. J Biol Chem 273: 7201-7204, 1998.

38. Gao X, Wang N, Wu S, Cui H, An X and Yang Y: Long noncoding RNA FER1L4 inhibits cell proliferation and metastasis through regulation of the PI3K/AKT signaling pathway in lung cancer cells. Mol Med Rep 20: 182-190, 2019. International (CC BY-NC-ND 4.0) License. 